\title{
Adaptive DWO Estimator of a Regression Function
}

Anatoli Juditski, Alexander Nazin, Jacob Roll, Lennart Ljung

Division of Automatic Control

E-mail: Anatoli.Iouditski@inrialpes.fr, nazine@ipu.rssi.ru, roll@isy.liu.se, ljung@isy.liu.se

14th June 2007

Report no.: LiTH-ISY-R-2794

Accepted for publication in Proc. NOLCOS 2004 - IFAC Symposium on Nonlinear Control Systems, Stuttgardt

Address:

Department of Electrical Engineering

Linköpings universitet

SE-581 83 Linköping, Sweden

WWW: http://wwW. control.isy.liu.se

AUTOMATIC CONTROL

REGLERTEKNIK

LINKÖPINGS UNIVERSITET

Technical reports from the Automatic Control group in Linköping are available from http://www. control.isy.liu.se/publications. 


\begin{abstract}
We address a problem of non-parametric estimation of an unknown regression function $f:[-1 / 2,1 / 2] \rightarrow R$ at a fixed point $x_{0} \in(-1 / 2,1 / 2)$ on the basis of observations $\left(x_{i}, y_{i}\right), i=1, \ldots, n$ such that $y_{i}=f\left(x_{i}\right)+e_{i}$, where $e_{i} \sim N\left(0, \sigma^{2}\right)$ is unobservable, Gaussian i.i.d. random noise and $x_{i} \in[-1 / 2,1 / 2]$ are given design points. Recently, the Direct Weight Optimization (DWO) method has been proposed to solve a problem of such kind. The properties of the method have been studied for the case when the unknown function $f$ is continuously differentiable with Lipschitz continuous derivative having a priori known Lipschitz constant $L$. The minimax optimality and adaptivity with respect to the design have been established for the resulting estimator. However, in order to implement the approach, both $L$ and $\sigma$ are to be known. The subject of the submission is the study of an adaptive version of the DWO estimator which uses a data-driven choice of the method parameter $L$.
\end{abstract}

Keywords: identification 


\title{
ADAPTIVE DWO ESTIMATOR OF A REGRESSION FUNCTION
}

\author{
Anatoli Juditsky* Alexander Nazin ${ }^{* *, 1}$ \\ Jacob Roll ${ }^{* * *}$ Lennart Ljung *** \\ * LMC-IMAG, BP 53, F-38041 Grenoble Cedex 9, France \\ Email: juditsky@inrialpes.fr \\ ** Institute of Control Sciences, Russian Acad. Sci., \\ Profsoyuznaya str., 65117997 Moscow, Russia \\ Email: nazine@ipu.rssi.ru \\ *** Div. of Automatic Control, Linkoping University \\ SE-58183 Linkoping, Sweden \\ Emails: roll, ljung@isy.liu.se
}

\begin{abstract}
We address a problem of non-parametric estimation of an unknown regression function $f:[-1 / 2,1 / 2] \rightarrow R$ at a fixed point $x_{0} \in(-1 / 2,1 / 2)$ on the basis of observations $\left(x_{i}, y_{i}\right), i=1, . ., n$ such that $y_{i}=f\left(x_{i}\right)+e_{i}$, where $e_{i} \sim N\left(0, \sigma^{2}\right)$ is unobservable, Gaussian i.i.d. random noise and $x_{i} \in[-1 / 2,1 / 2]$ are given design points. Recently, the Direct Weight Optimization (DWO) method has been proposed to solve a problem of such kind. The properties of the method have been studied for the case when the unknown function $f$ is continuously differentiable with Lipschitz continuous derivative having a priori known Lipschitz constant $L$. The minimax optimality and adaptivity with respect to the design have been established for the resulting estimator. However, in order to implement the approach, both $L$ and $\sigma$ are to be known. The subject of the submission is the study of an adaptive version of the DWO estimator which uses a data-driven choice of the method parameter $L$.
\end{abstract}

Copyright (C) 2004 IFAC

Keywords: Non-parametric regression, Estimators, Adaptive algorithms, Mean-square error, Quadratic programming

\section{INTRODUCTION}

Consistent non-parametric estimation of a regression function $f:[-1 / 2,1 / 2] \rightarrow R$ based on its noisy observations

$$
y_{i}=f\left(x_{i}\right)+e_{i}, \quad i=1, \ldots, n
$$

at some given design points $x_{i} \in(-1 / 2,1 / 2)$ is one of the basic problems for many applica-

1 Partially supported by the Swedish Royal Academy of Sciences via their research grant of 2003. tions including non-linear system identification, see, e.g., (Ljung, 1999). Here the random noise $e_{i}$ is supposed to be i.i.d. with $E e_{i}=0, E e_{i}^{2}=\sigma^{2}$, $\sigma>0$. A common approach to estimating $f\left(x_{0}\right)$ at a fixed point $x_{0} \in(-1 / 2,1 / 2)$ is to use a linear estimator

$$
\widehat{f}_{n}=\widehat{f}_{n}\left(x_{0}\right)=\sum_{i=1}^{n} w_{i} y_{i}
$$

The problem then reduces to finding a good vector $w=\left(w_{1}, \ldots, w_{n}\right)^{T}$ of weights $w_{i}=w_{i}\left(x_{0} ; X_{n}\right)$, $X_{n}=\left(x_{1}, \ldots, x_{n}\right)^{T}$, which gives a reasonably 
small Mean-Square Error (MSE)

$$
\operatorname{MSE}\left(\widehat{f}_{n}, f\right)=E\left[\left(\widehat{f}_{n}\left(x_{0}\right)-f\left(x_{0}\right)\right)^{2} \mid X_{n}\right]
$$

over a given function class $\mathcal{F}$.

A classic family of weights are generated by kernel methods, where a kernel function $K$ and a bandwidth $h_{n}$ are used to determine the weights. Another widely used approach is the local polynomial modelling approach, where the estimator is determined by locally fitting a polynomial to the given data; appropriate kernel and bandwidth should also be determined here. See, e.g., (Fan and Gijbels, 1996) for both the details and the references therein.

Recently, in (Roll et al., 2003a) and (Roll et al., 2003b), the Direct Weight Optimization (DWO) method has been proposed to solve a problem of considered type; note, that a similar approach has been earlier studied in (Sacks and Ylvisaker, 1978). The main idea of the DWO is to minimize the maximum MSE

$$
\mathcal{R}_{n}\left(\widehat{f}_{n}\right)=\sup _{f \in \mathcal{F}} M S E\left(\widehat{f}_{n}, f\right)
$$

or its "natural" upper bound $U_{n}(w)$. Particularly, the properties of the method have been studied for the case when the unknown function $f$ is continuously differentiable with Lipschitz continuous derivative having a priori known Lipschitz constant $L$. In this case, the upper bound on $\mathcal{R}_{n}\left(\widehat{f}_{n}\right)$ represents a convex quadratic function of $w \in R^{n}$ depending also on the parameters $\sigma$ and $L$. Moreover, it should be minimized subject to some linear constraints, and the problem reduces to a quadratic programming one (or to a cone program, in the multivariate case). The detailed study of the approach and simulation examples may be found in (Roll, 2003). Particularly, minimax optimality and adaptivity with respect to the design have been established (for non-negative weights).

However, in order to implement the approach, both $L$ and $\sigma$ are to be known. The goal of the submission is to propose and study an adaptive version of the DWO estimator which uses a datadriven choice of the method parameter $L$. It turns out that a payoff for the adaptivity to the unknown Lipschitz constant $L^{*}$ is expressed by a logarithmic factor to the MSE upper bound.

\section{PROBLEM STATEMENT}

Consider the problem of non-parametric estimation of the value $f\left(x_{0}\right)$ of an unknown function $f:[-1 / 2,1 / 2] \rightarrow R$ at a given point $x_{0} \in$ $(-1 / 2,1 / 2)$, given a set of input-output pairs $\left\{\left(x_{i}, y_{i}\right)\right\}_{i=1}^{n}$, coming from the relation

$$
y_{i}=f\left(x_{i}\right)+e_{i},
$$

where $e_{i} \sim N\left(0, \sigma^{2}\right)$ is unobservable, i.i.d. gaussian random noise and $x_{i} \in[-1 / 2,1 / 2]$ are given design points (non random, for the sake of simplicity); $\sigma>0$ is supposed to be a priori known. Function $f$ is continuously differentiable with Lipschitz continuous derivative

$$
\left|f^{\prime}(u)-f^{\prime}(v)\right| \leq L|u-v|
$$

with Lipschitz constant $L$ being a priori unknown; denote $\mathcal{F}(L)$ the (non-parametric) class of all functions meeting inequality (2). We consider the maximum Mean-square error (MSE)

$$
\mathcal{R}_{n}\left(\widehat{f}_{n}, L\right)=\sup _{f \in \mathcal{F}(L)} E_{f}\left\{\left(\widehat{f}_{n}\left(x_{0}\right)-f\left(x_{0}\right)\right)^{2}\right\}
$$

as a risk for an estimator $\widehat{f}_{n}$ over function class $\mathcal{F}(L)$. Here and further on, the expectation $E_{f}$ corresponds to the distribution of observations (1) generated by function $f$. Introduce

$$
\widetilde{x}_{i}=x_{i}-x_{0}
$$

For an arbitrary linear estimator

$$
\widehat{f}_{n}=\sum_{i=1}^{n} w_{i} y_{i}
$$

the following MSE Upper Bound holds true:

$$
\begin{aligned}
\mathcal{R}_{n}\left(\widehat{f}_{n}, L\right) & \leq U_{n}(w, L) \\
& =\left(\frac{L}{2} \sum_{i=1}^{n}\left|w_{i}\right| \widetilde{x}_{i}^{2}\right)^{2}+\sigma^{2}\|w\|^{2}
\end{aligned}
$$

with $\|\cdot\|$ standing for Euclidean norm. Note, that the last summand in the right hand side of (6) represents the variance and the first one the upper bound on the squared bias of the estimation error $\widehat{f}_{n}\left(x_{0}\right)-f\left(x_{0}\right)$.

The DWO approach to the given estimation problem with a priori known Lipschitz constant $L$ is to use estimator (5) with the weight vector $w=\left(w_{1}, \ldots, w_{n}\right)$ being a solution to the following optimization problem:

$$
U_{n}^{*}(L)=\min _{w \in R^{n}} U_{n}(w, L)
$$

subject to constraints

$$
\sum_{i=1}^{n} w_{i}=1, \quad \sum_{i=1}^{n} w_{i} \widetilde{x}_{i}=0 .
$$

Note, that computationally, this problem reduces to a quadratic program. Denote $w^{*}(n, L)$ the minimizer for $U_{n}(w, L)$ in the problem (7), (8). Thus, $U_{n}^{*}(L)=U_{n}\left(w^{*}(n, L), L\right)$. In what follows we extend the DWO approach to the case of unknown Lipschitz constant $L$. 


\section{ADAPTIVE DWO ESTIMATOR}

Suppose that it is known a priori that $U_{n}^{*}\left(L^{*}\right) \in$ $\left[U_{n}^{\min }, U_{n}^{\max }\right] \subset(0, \infty)$ where $L^{*}$ stands for "true Lipschitz constant" of $f^{\prime}(x)$. Let us fix $\alpha \in(0,1)$ and a related integer $K=K(\alpha)>1$. Consider the decreasing sequence $U=\left(U_{k}\right), k=1, \ldots, K$, such that $U_{1}=U_{n}^{\max }, U_{K}=U_{n}^{\min }$, and

$$
U_{k} / U_{k-1} \leq \alpha<1, \quad k=2, \ldots, K .
$$

Note that as $U_{n}^{*}(L)$ is monotonously increasing in $L$, for any $U_{k}$ one can easily find $L_{k}$ (e.g., by bisection) which gives $U_{n}^{*}\left(L_{k}\right)=U_{k}$. Thus, $L_{\max }=L_{1}$ stands for maximum Lipschitz constant $L$. Note, that typically

$$
U_{n}^{\max }=O\left(\sigma^{2}\right)
$$

and

$$
U_{n}^{\min }=O\left(\sigma^{2} \frac{\sum_{i=1}^{n} \widetilde{x}_{i}^{2}}{n \sum_{i=1}^{n} \widetilde{x}_{i}^{2}-\left(\sum_{i=1}^{n} \widetilde{x}_{i}\right)^{2}}\right) .
$$

For each couple $(i, k)$ such that $2 \leq i \leq K$ and $1 \leq k<i$ introduce

$$
s_{i k}=\sigma_{i k} \sqrt{2 \ln \frac{U_{i}}{U_{n}^{\min }}}+2 b_{i}
$$

with $\sigma_{i k}=\sigma\left\|w^{(k)}-w^{(i)}\right\|$ and

$$
b_{i}=\frac{L_{i}}{2} \sum_{j=1}^{n} \widetilde{x}_{j}^{2}\left|w_{j}^{(i)}\right| .
$$

Consider the following (adaptive DWO) algorithm the idea of which arises from (Lepski, 1990).

1 For each $U_{k} \in U, k=1, \ldots, K$ compute the corresponding $L_{k}$ and the vector

$$
w^{(k)}=w^{*}\left(n, L_{k}\right) .
$$

Form the related auxiliary estimates

$$
\widehat{f}_{n}^{(k)}=\sum_{i=1}^{n} w_{i}^{(k)} y_{i}, \quad k=1, \ldots, K .
$$

2 The adaptive estimate $\widehat{f}_{n}$ is defined as follows: We call $i, 1 \leq i \leq K$, admissible if

$$
\widehat{f}_{n}^{(i)} \in \bigcap_{1 \leq k<i}\left[\widehat{f}_{n}^{(k)}-s_{i k}, \widehat{f}_{n}^{(k)}+s_{i k}\right] .
$$

Clearly, an admissible $i$ exists, e.g. $i=1$ (because intersection over an empty index set equals $R$ ). Then we set $\widehat{i}$ the largest of admissible $i$ :s and put $\widehat{f}_{n}=\widehat{f}_{n}^{(\widehat{i})}$.

This concludes the algorithm.

\section{MAIN RESULTS}

Theorem 1. Consider the adaptive estimator $\widehat{f}_{n}$ defined in the previous section. Let the parameter $\alpha \in(0,1)$ be fixed. There exists such an absolute constant $C(\alpha)<\infty$ that for any $f \in \mathcal{F}\left(L_{\max }\right)$ the MSE for a defined above adaptive estimator meets the inequality

$$
\begin{aligned}
& E_{f}\left(\widehat{f_{n}}-f\left(x_{0}\right)\right)^{2} \\
& \leq C(\alpha)\left[U_{n}^{*}\left(L^{*}\right)\left(\ln \frac{U_{n}^{*}\left(L^{*}\right)}{U_{n}^{\min }}+1\right)\right. \\
& \left.\quad+U_{n}^{\min } \ln \frac{U_{n}^{\max }}{U_{n}^{*}\left(L^{*}\right)}\right] .
\end{aligned}
$$

Note, that the rough bounds on $U_{n}^{\min }$ and $U_{n}^{\max }$ can always be taken as follows (see Appendix B for their proof):

$$
U_{n}^{\min }=\frac{\sigma^{2}}{n}, \quad U_{n}^{\max }=\left(\frac{L_{\max }}{2}\right)^{2}+\sigma^{2} ;
$$

the last expression for $U_{n}^{\max }$ is proved when

$$
\min _{1 \leq i \leq n} \widetilde{x}_{i}<0<\max _{1 \leq i \leq n} \widetilde{x}_{i} .
$$

With these bounds on risk, one obtains

$$
\ln \left(U_{n}^{\max } / U_{n}^{\min }\right)=\ln n+O(1), n \rightarrow \infty
$$

Hence, $\ln \left(U_{n}^{*}\left(L^{*}\right) / U_{n}^{\min }\right)$ and $\left.\ln \left(U_{n}^{\max }\right) / U_{n}^{*}\left(L^{*}\right)\right)$ are of the order $O(\ln n)$. Thus, we arrive at the following asymptotic result.

Corollary 2. Under the assumptions of Theorem 1

$$
\limsup _{n \rightarrow \infty} \frac{E_{f}\left(\widehat{f}_{n}-f\left(x_{0}\right)\right)^{2}}{U_{n}^{*}\left(L^{*}\right) \ln n} \leq C(\alpha) .
$$

Note, that typically $U_{n}^{*}\left(L^{*}\right)=O\left(n^{-\nu}\right)$ with $\nu>0$ depending on the design. For instance, equidistant design leads to $\nu=4 / 5$, see (Roll, 2003) for the details.

\section{CONCLUSION}

The adaptive DWO algorithm assumes upper bound on Lipschitz constant $L_{\max } \geq L^{*}$ being known a priori. However, even when this bound is very large, the adaptive method will attain the same MSE (up to the log factor) as the unimplementable DWO estimator, which "knows" the exact Lipschitz constant. On the other hand, if the non-adaptive DWO estimator is used with the upper bound $L_{\max }$, its performance will degrade severely if $L^{*}<<L_{\max }$. 
Furthermore, it can be shown that the logarithmic loss in the accuracy with respect to the unimplementable ("oracle") algorithm, which attains the MSE $U_{n}^{*}\left(L^{*}\right)$, in a certain sense, cannot be suppressed using any estimation procedure, and represents an unavoidable price for the lack of the prior knowledge of $L^{*}$.

Acknowledgements The authors would like to thank Oleg Lepski for the fruitful discussion. The second author also thanks the Swedish Research Council (VR) for their support.

\section{REFERENCES}

Fan, J. and I. Gijbels (1996). Local Polynomial Modelling and Its Applications. Chapman \& Hall.

Lepski, O.V. (1990). One problem of adaptive estimation in gaussian white noise. Theory Probab. Appl. 35, 454-466.

Ljung, L. (1999). System Identification: Theory For the User, 2nd ed,. Prentice Hall. Upper Saddle River, N.J.

Roll, J., A. Nazin and L. Ljung (2003a). Local modelling of nonlinear dynamic systems using direct weight optimization. In: 13th IFAC Symposium on System Identification. Rotterdam. pp. 1554-1559.

Roll, J., A. Nazin and L. Ljung (2003b). Local modelling with a priori known bounds using direct weight optimization. In: European Control Conference. Cambridge.

Roll, Jacob (2003). Local and Piecewise Affine Approaches to System Identification. $\mathrm{PhD}$ thesis. Linköping University.

Sacks, J. and D. Ylvisaker (1978). Linear estimation for approximately linear models. Ann. Statist. 6(5), 1122-1137.

\section{APPENDIX A}

Below is a sketch of the proof for Theorem 1.

1) First we note that for $1 \leq k<i \leq K$

$$
\begin{aligned}
& \left|\widehat{f}_{n}^{(k)}-\widehat{f}_{n}^{(i)}\right| \\
= & \left|\sum_{j=1}^{n}\left(w_{j}^{(k)}-w_{j}^{(i)}\right) f\left(x_{j}\right)+\sum_{j=1}^{n}\left(w_{j}^{(k)}-w_{j}^{(i)}\right) e_{j}\right| \\
\leq & \left|\sum_{j=1}^{n}\left(w_{j}^{(k)}-w_{j}^{(i)}\right)\left(f\left(x_{j}\right)-f\left(x_{0}\right)-f^{\prime}\left(x_{0}\right) \widetilde{x}_{j}\right)\right| \\
& +\left|\sum_{j=1}^{n}\left(w_{j}^{(k)}-w_{j}^{(i)}\right) e_{j}\right|
\end{aligned}
$$

$\leq \frac{L^{*}}{2} \sum_{j=1}^{n}\left(\left|w_{j}^{(k)}\right|+\left|w_{j}^{(i)}\right|\right) \widetilde{x}_{j}^{2}+\left|\xi_{i k}\right| \sigma_{i k}$,

where $\xi_{i k} \sim N(0,1)$.

Further, for any $1 \leq i, k \leq K$

$$
b_{i}^{2}+\sigma^{2}\left\|w^{(i)}\right\|^{2} \leq \frac{L_{i}^{2}}{L_{k}^{2}} b_{k}^{2}+\sigma^{2}\left\|w^{(k)}\right\|^{2}
$$

(as $w^{(i)}$ is the minimizer which corresponds to $L_{i}$ ), so that (by summing the $i$ 's and $k$ 's inequalities above)

$$
b_{i}^{2}+b_{k}^{2} \leq \frac{L_{i}^{2}}{L_{k}^{2}} b_{k}^{2}+\frac{L_{k}^{2}}{L_{i}^{2}} b_{i}^{2},
$$

and, for $i>k$, as $L_{k}>L_{i}$, one obtains

$$
b_{k} \leq \frac{L_{k} b_{i}}{L_{i}} .
$$

2) Let $i^{*}$ be such that

$$
U_{n}^{*}\left(L^{*}\right) \leq U_{i^{*}} \leq \alpha^{-1} U_{n}^{*}\left(L^{*}\right)
$$

Consider first the case $\widehat{i} \geq i^{*}$. Then due to the admissibility of $\widehat{i}$ and inequality $L^{*} \leq L_{i^{*}}$,

$$
\begin{aligned}
& \left.E\left|\widehat{f}_{n}-f\left(x_{0}\right)\right|^{2} 1 \hat{i} \geq i^{*}\right\} \\
\leq & 2 E\left|\widehat{f}_{n}-\widehat{f}_{n}^{i^{*}}\right|^{2} 1\left\{\hat{i} \geq i^{*}\right\} \\
& +2 E\left|\widehat{f}_{n}^{i^{*}}-f\left(x_{0}\right)\right|^{2} \leq 2 E s_{i_{i i^{*}}^{2}}^{2}+2 U_{n}\left(w^{\left(i^{*}\right)}, L^{*}\right) \\
\leq & 4 E\left[2 \sigma_{\hat{i i^{*}}}^{2} \ln \left(\frac{U_{\hat{i}}}{U_{n}^{\text {min }}}\right)+4 b_{\hat{i}}^{2}\right]+2 U_{i^{*}} .
\end{aligned}
$$

(Here and further on we use a simpler notation $E$ for the expectation $E_{f}$ assuming function $f$ is fixed.) On the other hand, since $U_{\hat{i}} \leq U_{i^{*}}$ and $\left\|w^{(k)}-w^{(i)}\right\|^{2} \leq 2\left\|w^{(k)}\right\|^{2}+2\left\|w^{(i)}\right\|^{2}$, we have

$$
\begin{aligned}
& \sigma_{\hat{i}^{*}}^{2} \ln \left(\frac{U_{\hat{i}}}{U_{n}^{\min }}\right)+2 b_{\hat{i}}^{2} \\
\leq & 2\left[\sigma^{2}\left\|w^{(\widehat{i})}\right\|^{2} \ln \left(\frac{U_{\hat{i}}}{U_{n}^{\min }}\right)+b_{\hat{i}}^{2}\right] \\
& +2 \sigma^{2}\left\|w^{\left(i^{*}\right)}\right\|^{2} \ln \left(\frac{U_{\widehat{i}}}{U_{n}^{\min }}\right) \\
\leq & C U_{i^{*}} \ln \left(\frac{U_{i^{*}}}{U_{n}^{\min }}\right)
\end{aligned}
$$

with some finite constant $C$. Thus

$$
\begin{aligned}
& E\left|\widehat{f}_{n}-f\left(x_{0}\right)\right|^{2} 1\left\{\hat{i} \geq i^{*}\right\} \\
\leq & 8\left(C \ln \left(\frac{U_{i^{*}}}{U_{n}^{\min }}\right)+1\right) U_{i^{*}}
\end{aligned}
$$

(a finer estimate can probably be obtained). We now aim to upper bound $E\left|\widehat{f}_{n}-f\left(x_{0}\right)\right|^{2} 1\left\{\hat{i}<i^{*}\right\}$. To this end for $1 \leq k<i \leq i^{*}$ we use (13): 


$$
\begin{aligned}
& \left|\widehat{f}_{n}^{(i)}-\widehat{f}_{n}^{(k)}\right| \\
\leq & \frac{L^{*}}{2} \sum_{j=1}^{n}\left(\left|w_{j}^{(k)}\right|+\left|w_{j}^{(i)}\right|\right) \widetilde{x}_{j}^{2} \\
& +\left|\xi_{i k}\right| \sigma_{i k} \\
= & \frac{L^{*}}{L_{k}} b_{k}+\frac{L^{*}}{L_{i}} b_{i}+\left|\xi_{i k}\right| \sigma_{i k} \\
\left(\text { as } i^{*} \geq i>k\right) \leq & \frac{L_{i}}{L_{k}} b_{k}+b_{i}+\left|\xi_{i k}\right| \sigma_{i k} \\
(\text { by (14) }) \leq & 2 b_{i}+\left|\xi_{i k}\right| \sigma_{i k} .
\end{aligned}
$$

Thus $\widehat{i}<i^{*}$ only if for some $k<i \leq i^{*},\left|\xi_{i k}\right|>$ $\sqrt{2 \ln \left(U_{i} / U_{n}^{\min }\right)}$. Further, since $\xi_{i k}$ has standard Gaussian distribution one may prove from Lemma 3 the inequality

$$
\begin{aligned}
& E\left(\sum_{j=1}^{n} e_{j} w_{j}^{(i)}\right)^{2} 1\left\{\left|\xi_{i k}\right|>\lambda\right\} \\
& \quad \leq \sqrt{\frac{2}{\pi}}\left(\lambda+\lambda^{-1}\right) \sigma^{2}\left\|w^{(i)}\right\|^{2} e^{-\lambda^{2} / 2}
\end{aligned}
$$

and for $\lambda=\sqrt{2 \ln \left(U_{i} / U_{n}^{\min }\right)}$ this expectation $\leq C \sqrt{\ln \left(U_{i} / U_{n}^{\min }\right)} \sigma^{2}\left\|w^{(i)}\right\|^{2} U_{n}^{\min } / U_{i}$.

Now, since $\widehat{f}_{n}=\widehat{f}_{n}^{(\widehat{i})}$, one obtains

$$
\begin{aligned}
& \quad\left|\widehat{f}_{n}^{(\widehat{i})}-f\left(x_{0}\right)\right| 1\left\{\widehat{i}<i^{*}\right\} \\
& \leq \sum_{i<i^{*}}\left(\left|E \widehat{f}_{n}^{(i)}-f\left(x_{0}\right)\right|\right. \\
& \left.\quad+\left|\widehat{f}_{n}^{(i)}-E \widehat{f}_{n}^{(i)}\right|\right) 1\{\widehat{i}=i\}
\end{aligned}
$$

and, for $i<i^{*}$, by (14)

$$
\left|E \widehat{f}_{n}^{(i)}-f\left(x_{0}\right)\right| \leq \frac{L^{*}}{L_{i}} b_{i} \leq b_{i^{*}}
$$

and, by (17) and $i^{*} \leq C \ln \left(U_{n}^{\max } / U_{i^{*}}\right)$,

$$
\begin{aligned}
& E\left|\widehat{f}_{n}^{(i)}-E \widehat{f}_{n}^{(i)}\right|^{2} 1\{\widehat{i}=i\} \\
\leq & \sum_{k<i} E\left(\sum_{j=1}^{n} e_{j} w_{j}^{(i)}\right)^{2} 1\left\{\left|\xi_{i k}\right|>\lambda\right\} \\
\leq & C i \sigma^{2}\left\|w^{(i)}\right\|^{2} \frac{U_{n}^{\min }}{U_{i}} \leq C U_{n}^{\min } \ln \frac{U_{n}^{\max }}{U_{i^{*}}} .
\end{aligned}
$$

Thus, the bounds (18)-(20) lead to

$$
\begin{aligned}
& \quad E\left|\widehat{f}_{n}^{(\widehat{i})}-f\left(x_{0}\right)\right|^{2} 1\left\{\widehat{i}<i^{*}\right\} \\
& \leq 2 \sum_{i<i^{*}} E\left(\left|E \widehat{f}_{n}^{(i)}-f\left(x_{0}\right)\right|^{2}\right. \\
& \left.\quad+\left|\widehat{f}_{n}^{(i)}-E \widehat{f}_{n}^{(i)}\right|^{2}\right) 1\{\widehat{i}=i\}
\end{aligned}
$$

$$
\leq 2 b_{i^{*}}^{2}+C U_{n}^{\min } \ln \frac{U_{n}^{\max }}{U_{i^{*}}}
$$

3) Finally, combining the upper bounds (16), (21) and using (15) we arrive at the result of the Theorem.

Lemma 3. Let the random variables $\xi$ and $\eta$ be Gaussian with $E \xi=E \eta=0, E \xi^{2}=E \eta^{2}=1$. Then, for any non-random $\lambda>0$ the following inequality holds

$$
E\left(\eta^{2} 1\{|\xi|>\lambda\}\right) \leq \sqrt{\frac{2}{\pi}}\left(\lambda+\lambda^{-1}\right) e^{-\lambda^{2} / 2} .
$$

\section{APPENDIX B}

The bounds (10) are proved as follows. By definition (6), the solution to minimization problem (7)-(8) is bounded by

$$
U_{n}(w, L) \geq \sigma^{2} \min _{w^{T} \mathbf{1}_{n}=1}\|w\|^{2}=\frac{\sigma^{2}}{n}
$$

where $\mathbf{1}_{n}=(1,1, \ldots, 1)^{T} \in R^{n}$. Similarly,

$$
U_{n}^{*}\left(L^{*}\right) \leq U_{n}^{*}\left(L_{\max }\right) \leq U_{n}\left(w^{+}, L_{\max }\right)
$$

where weight vector $w^{+}$has non-negative entries and meets constraints (8), existing due to (11). Thus, second equality (10) follows from (24) due to $\left|\widetilde{x}_{i}\right| \leq 1$ and $\left\|w^{+}\right\| \leq 1$. The bounds (10) are proved. 


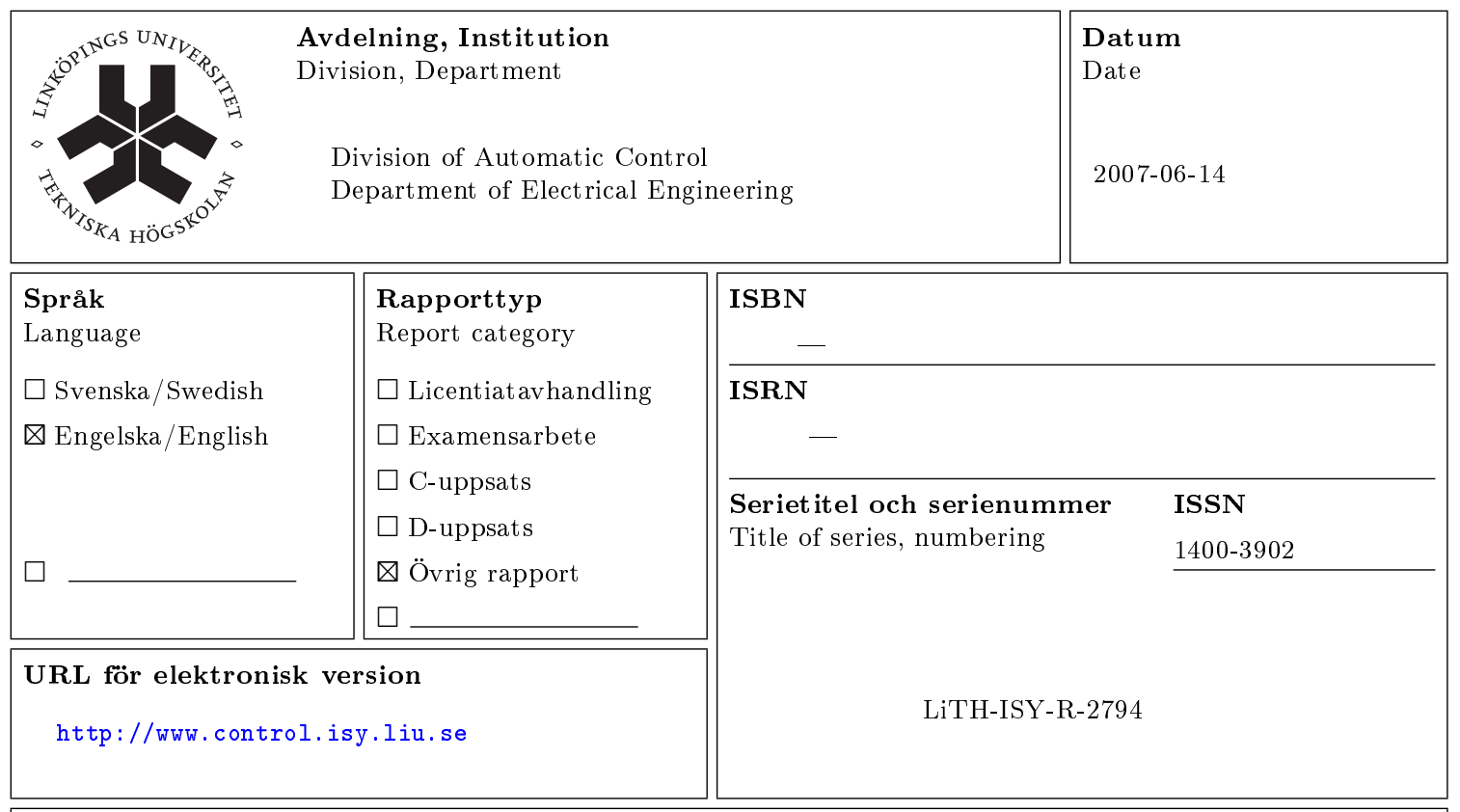

Titel Adaptive DWO Estimator of a Regression Function

Title

Adaptive DWO Estimator of a Regression Function

Författare Anatoli Juditski, Alexander Nazin, Jacob Roll, Lennart Ljung

Author

Sammanfattning

Abstract

We address a problem of non-parametric estimation of an unknown regression function $f$ : $[-1 / 2,1 / 2] \rightarrow R$ at a fixed point $x_{0} \in(-1 / 2,1 / 2)$ on the basis of observations $\left(x_{i}, y_{i}\right)$, $i=1, . ., n$ such that $y_{i}=f\left(x_{i}\right)+e_{i}$, where $e_{i} \sim N\left(0, \sigma^{2}\right)$ is unobservable, Gaussian i.i.d. random noise and $x_{i} \in[-1 / 2,1 / 2]$ are given design points. Recently, the Direct Weight Optimization (DWO) method has been proposed to solve a problem of such kind. The properties of the method have been studied for the case when the unknown function $f$ is continuously differentiable with Lipschitz continuous derivative having a priori known Lipschitz constant $L$. The minimax optimality and adaptivity with respect to the design have been established for the resulting estimator. However, in order to implement the approach, both $L$ and $\sigma$ are to be known. The subject of the submission is the study of an adaptive version of the DWO estimator which uses a data-driven choice of the method parameter $L$. 